\title{
Mechanosensitive Osteogenesis on Native Cellulose Scaffolds for Bone Tissue Engineering.
}

\author{
Maxime Leblanc Latour ${ }^{1}$ and Andrew E. Pelling ${ }^{1,2,3}$ \\ ${ }^{1}$ Department of Physics, STEM Complex, 150 Louis Pasteur, University of Ottawa, Ottawa, ON, \\ K1N 6N5, Canada \\ ${ }^{2}$ Department of Biology, Gendron Hall, 30 Marie Curie, University of Ottawa, Ottawa, ON, K1N \\ $6 \mathrm{~N} 5$, Canada \\ ${ }^{3}$ Institute for Science, Society and Policy, Desmarais Building, 55 Laurier Ave. East, University \\ of Ottawa, Ottawa, ON, K1N 6N5, Canada
}

Author for correspondence: $\underline{\text { a@pellinglab.net. }}$

\begin{abstract}
In recent years, plant-derived cellulosic biomaterials have become a popular way to create scaffolds for a variety of tissue engineering applications. Moreover, such scaffolds possess similar physical properties (porosity, stiffness) that resemble bone tissues and have been explored as potential biomaterials for tissue engineering applications. Here, plant-derived cellulose scaffolds were seeded with MC3T3-E1 pre-osteoblast cells. Moreover, to assess the potential these biomaterials we also applied cyclic hydrostatic pressure (HP) to the cells and scaffolds over time to mimic a bone-like environment more closely. After one week of proliferation, cell-seeded scaffolds were exposed to $\mathrm{HP}$ up to $270 \mathrm{KPa}$ at a frequency of $1 \mathrm{~Hz}$, once per day, for up to two weeks. Scaffolds were incubated in osteogenic inducing media (OM) or regular culture media (CM). The effect of cyclic HP combined with OM on cell-seeded scaffolds resulted in an increase of differentiated cells. This corresponded with an upregulation of alkaline phosphatase activity and scaffold mineralization. The results reveal that in vitro, the mechanosensitive pathways which regulate osteogenesis appear to be functional on novel plant-derived cellulosic biomaterials.
\end{abstract}

\section{Introduction}

Upon injury or break, bone has the ability to self-renew. However, large defects created by either injury or disease may require graft placement to avoid non-union or malunion of the bone tissue (Andrzejowski and Giannoudis, 2019). Such grafts can be derived directly from the patient (autologous grafts), and is considered the "gold standard" in regenerative orthopedics (Campana et al., 2014; Parikh, 2002; Sakkas et al., 2017; Wang and Yeung, 2017). However, limited size grafts, donor site morbidity and infections, cost and post-operative pain at both donor and receiver site has led to the development of alternative approaches (Parikh, 2002; Wang and Yeung, 2017): cadaver donors (allograft), animal sources (xenograft), or artificially derived (alloplastic). Such alternatives all have their own benefits and drawbacks, the later however provides a potential alternative with lower risk of transmitted diseases and infections, as well as overcoming the size limitation barrier (Parikh, 2002; Wang and Yeung, 2017) . 
Alloplastic grafts are also considered a more ethical alternative to allografts and xenografts (Fernández et al., 2015). Physical properties are key parameters for graft development, such as pore size, interconnectivity and elasticity (Campana et al., 2014; Gao et al., 2019; Nukavarapu et al., 2015). Fine tuning of these parameters leads to better mechanical support and stability of the implant.

Long bones are highly dynamic structural tissues. A whole spectrum of forces are acting on different areas of this part of the skeletal system. For instance, the pressure found in the femur head in human adults can reach $5 \mathrm{MPa}$ during normal locomotion, and can reach up to $18 \mathrm{MPa}$ for other activities (Morrell et al., 2005). On a microscopic level, these forces are transmitted to the osteocytes through Wnt/ $\beta$-catenin mechano-sensing pathways in the lacuna-canaliculi network (Bonewald and Johnson, 2008). Force-regulated mechanisms lead to formation and removal of bone tissue through bone remodeling processes (Bonewald and Johnson, 2008) and the pressure inside the lacuna-canaliculi network is around $280 \mathrm{kPa}$ (Zhang et al., 1998). Bioreactors have also been developed to apply stresses to osteoblast cells to replicate the native bone environment via uniaxial compression/tension, biaxial compression/tension, shearstress, etc (Brunelli et al., 2019; Pörtner et al., 2005). Hydrostatic pressure (HP) stimulation on cultured cells has also been achieved by compressing the gas phase above an incompressible media (Gardinier et al., 2009; Henstock et al., 2013; Liu et al., 2010; Reinwald et al., 2015; Reinwald and El Haj, 2018; Stavenschi et al., 2018; Zhao et al., 2015). Three-dimensional (3D) culturing of the cells is also critical for better representing in vivo conditions. With a specific tissue-oriented scaffold structure and appropriate applied mechanical stimuli one can potentially predict the performance of a biomaterial scaffold prior to in vivo animal studies.

A variety of $3 \mathrm{D}$ biomaterials have been utilized support the growth and proliferation of cells in environments that mimic bone tissues. These include hydroxyapatite, tricalcium phosphate, bioceramics or bioactive glass (Wang and Yeung, 2017). These materials are osteoconductive, can promote osteointegration and provide structural support at the implant site (Wang and Yeung, 2017). However, they show poor osteoconduction and little osteogenesic response (Wang and Yeung, 2017). Polymer biomaterials such as poly(glycolic acid) (PGA), poly(lactic

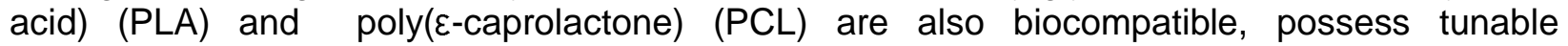
degradation rates and can be chemically modify to change the surface chemistry (Wei et al., 2020). However, in vivo degradation creates acidic bi-products which can lead to an inflammatory response and decrease the efficiency bone repair (Wei et al., 2020). Finally, naturally occurring polymers such as collagen, chitosan and silk are also common (Wei et al., 2020). However, due to their inherent mechanical properties and structural stability, these materials are often utilized as coipmosites with additional polymers and coatings in BTE applications (Di Martino et al., 2005; Lee and Volpicelli, 2017; Wei et al., 2020) . More recently, plant-derived decellularized cellulose scaffolds have been shown to be effective in BTE applications (Hickey and Pelling, 2019; Lee et al., 2019; Torgbo and Sukyai, 2018).

Previous studies by our group and others have shown that cellulose based scaffolds derived from plants can be used as tissue engineering scaffolds (Gershlak et al., 2017; Hickey et al., 2018; Lee et al., 2019; Modulevsky et al., 2016, 2014). These biomaterials are often sourced from plants with a microstructure that closely mimics the tissue to be replicated (Hickey et al., 2018). Successful experiments in vitro and in vivo showed that these biomaterials are biocompatible and support angiogenesis (Hickey et al., 2018; Modulevsky et al., 2016, 2014). Due to their pore size interconnectivity, other groups have successfully differentiated human pluripotent stem cells into bone-like tissues within scaffolds derived from either decellularized mushrooms (Balasundari et al., 2012) or apple tissues (Lee et al., 2019). The in vivo performance of the apple-derived scaffolds were further examined by implanting disk-shaped 
scaffolds in rat cranial defects (Lee et al., 2019). The findings demonstrate partial bone regeneration within the implant, type 1 collagen deposition and blood vessel formation (Lee et al., 2019). However, to date, the mechanobiology of cells cultured on plant-derived scaffolds has not been examined. It remains poorly understood how mechanical signal transduction pathways are, or are not, impacted when cultured on unconventional plant-derived cellulosic biomaterials. Here, to further examine the potential role plant-based biomaterials can play in BTE applications, we explore how mechanical stimulation impacts the differentiation of preosteoblasts when cultured on plant cellulose scaffolds. In a custom-built bioreactor, we apply cyclic HP stimulation to differentiating osteoblasts and examine the regulation of key markers of osteogenesis and mineralization. The results reveal that application of HP, in combination with osteogenic inducing media, leads to enhanced differentiation. This work provides further evidence that plant-derived cellulose scaffolds support osteogenesis and have potential applications in BTE.

\section{Materials and Methods}

\section{Scaffold fabrication}

Samples were prepared following established protocols (Hickey et al., 2018; Modulevsky et al., 2016, 2014). Maclntosh apples (Canada Fancy) were cut with a mandolin slicer to $1 \mathrm{~mm}$-thick slices. A biopsy punch was used to create $5 \mathrm{~mm}$-diameter disks in the hypanthium tissue of the apple slices. The disks were decellularized in a $0.1 \%$ sodium dodecyl sulfate solution (SDS, Fisher Scientific, Fair Lawn, NJ) for two days. Then, the decellularized disks were washed in deionized water, before incubation in $100 \mathrm{mM} \mathrm{CaCl}_{2}$ for two days. The samples were sterilized with $70 \%$ ethanol, washed in deionized water, and placed in a 96-well culture plate prior to cell seeding.

MC3T3-E1 Subclone 4 cells (ATCC® CRL-2593 ${ }^{\mathrm{TM}}$, Manassas, VA) were cultured and maintained in a humidified atmosphere of $95 \%$ air and $5 \% \mathrm{CO}_{2}$, at $37^{\circ} \mathrm{C}$. The cells were cultured in Minimum Essential Medium (ThermoFisher, Waltham, MA), supplemented with 10\% Fetal Bovine Serum (Hyclone Laboratories Inc., Logan, UT) and 1\% Penicillin/Streptomycin (Hyclone Laboratories Inc). Scaffolds were placed in 96-well plates. Prior to cell seeding, scaffolds were immersed in culture media and incubated in a humidified atmosphere of $95 \%$ air and $5 \% \mathrm{CO}_{2}$, at $37^{\circ} \mathrm{C}$, for $30 \mathrm{~min}$. The culture media was completely aspirated from the wells. Cells were suspended and a $30 \mu \mathrm{L}$ drop containing $5 \times 10^{4}$ cells, was pipetted on each scaffold. The cells were left to adhere on the scaffolds for 2 hours before adding $200 \mu \mathrm{L}$ of culture media to the culture wells. Culture media was then changed every 3-4 days for 1 week. Cell seeded scaffolds were then either incubated in osteogenic media (OM) by adding $50 \mu \mathrm{g} / \mathrm{mL}$ of ascorbic acid and $10 \mathrm{mM} \beta$-glycerophosphate to the culture media or incubated in culture media (CM) for 2 weeks, with or without the application of HP.

\section{Cyclic hydrostatic pressure stimulation}

Cyclic HP was applied by modulating the pressure in the gas phase above the culture wells in a custom-build pressure chamber (Figure 1, A). The humidified, 95\% air and $5 \% \mathrm{CO}_{2}$ incubator atmosphere was compressed using an air compressor and injected in the chamber. A Particle Photon microcontroller (Particle Industries, San Francisco, CA) was used to control the frequency of the applied pressure remotely via a custom-made cellphone application through the Blynk loT platform (Blynk, New York, NY)). Cyclic HP was applied 1 hour per day, for up to 2 
weeks (Figure 1, B) at a frequency $1 \mathrm{~Hz}$, oscillating between 0 and $280 \mathrm{kPa}$ with respect to ambient pressure. Pressure was monitored using a pressure transducer. The samples were removed from the pressure chamber after each cycle and kept at ambient pressure between the stimulation phases.

Cell-seeded scaffolds were either stimulated with cyclic HP with and without the presence of OM, leading to four experimental conditions (Figure 1, B): Cyclic HP in regular culture media (CM-HP), cyclic HP in osteogenic culture media (OM-HP), non-stimulated in osteogenic media $(\mathrm{OM}-\mathrm{CTRL})$ and non-stimulated in regular culture media (CM-CTRL). The OM-CTRL and CMCTRL conditions were in a humidified, $5 \% \mathrm{CO} 2$ incubator at $37^{\circ} \mathrm{C}$.

\section{Scaffold imaging}

After 1 week or 2 weeks, scaffolds were washed with PBS and fixed with $10 \%$ neutral buffered formalin for $10 \mathrm{~min}$. Scaffolds were washed with PBS and incubated in a $0.01 \%$ Congo Red staining solution (Sigma-Aldrich, St. Louis, MO) for $20 \mathrm{~min}$ at room temperature. Scaffolds were washed with PBS. Cell nuclei were stained with 1:1000 Hoechst (ThermoFisher, Waltham, MA) for 30 min in the dark. Samples were washed with PBS and stored in wash buffer solution (5\% FBS in PBS) prior to imaging. The cell-seeded surface of the scaffolds was imaged with a laser scanning confocal microscope (Nikon Ti-E A1-R) equipped with a 10X objective. Maximum intensity projections were used for cell counting with ImageJ software (Schindelin et al., 2012). Cells were counted on a 1.3 by $1.3 \mathrm{~mm}^{2}$ area $(\mathrm{N}=3$ per experimental conditions with 3 randomly selected area per scaffold).

\section{Alkaline phosphatase activity assay}

Alkaline phosphatase (ALP) activity in media was measured using an ALP assay kit (BioAssay Systems, Hayward, CA). Working solution was prepared with $5 \mathrm{mM}$ magnesium acetate and 10 $\mathrm{mM}$ p-nitrophenyl phosphate (pNPP) in assay buffer, following manufacturer's protocol. $150 \mu \mathrm{L}$ of working solution was pipetted in 96-well plate. $200 \mu \mathrm{L}$ of calibrator solution and $200 \mu \mathrm{L}$ of $\mathrm{dH}_{2} \mathrm{O}$ were pipetted in separated well, in the same 96-well plate. At 1 week and 2 weeks, $20 \mu \mathrm{L}$ of incubation media was pipetted into the working solution's well. All wells were read at $405 \mathrm{~nm}$ for 10 minutes, every 30 seconds. ALP activity was calculated by taking the slope of the $405 \mathrm{~nm}$ readings vs time, calibrated with the calibrator solution and $\mathrm{dH}_{2} \mathrm{O}$. Wells were read in triplicates ( $\mathrm{N}=3$ per experimental conditions).

\section{Alizarin red S staining and mineral deposit quantification}

Samples were fixed with $10 \%$ neutral buffered formalin for $10 \mathrm{~min}$, after 1 week or 2 weeks. Calcium quantification was performed using previously published protocol (Gregory et al., 2004). Samples were transferred to a 24-well plate and carefully washed with deionized water and incubated in $1 \mathrm{~mL}$ of $40 \mathrm{mM}(\mathrm{pH}=4.1$ ) alizarin red $\mathrm{s}$ (ARS, Sigma-Aldrich) solution for 20 minutes at room temperature, with light agitation. The samples were washed with deionized

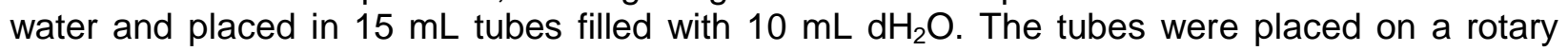
shaker at $120 \mathrm{rpm}$ for $60 \mathrm{~min}$ and $\mathrm{dH}_{2} \mathrm{O}$ was replaced every $15 \mathrm{~min}$. Thereafter, samples were incubated in $800 \mu \mathrm{L}$ of $10 \%$ acetic acid on an orbital shaker at $60 \mathrm{rpm}$ for $30 \mathrm{~min}$. The eluted ARS/acetic acid solution was pipettes out of the well and transferred to $1.5 \mathrm{~mL}$ centrifuge tubes. Tubes were centrifuged at $17 \times 10^{4} \mathrm{~g}$ for $15 \mathrm{~min}$. $500 \mu \mathrm{L}$ of supernatants were transferred to new centrifuge tube and $200 \mu \mathrm{L}$ of $10 \%$ ammonium hydroxide was pipetted into the tubes. Finally, $150 \mu \mathrm{L}$ of the solution was pipetted into a 96-well plate and the absorption at $405 \mathrm{~nm}$ 
was read using a plate reader (BioTek Instruments, Winooski, VT). Wells were read in triplicates ( $\mathrm{N}=3$ per experimental conditions).

\section{Statistical analysis}

Values reported in this manuscript are the average value \pm standard error of the mean (SEM). Statistical significance was determined using one-way ANOVA and post hoc Tukey test. A value of $p<0.05$ was considered to be statistically significant.

\section{Results}

\section{Scaffold imaging and cell counting}

Mechanical stimulation of the cultured scaffolds was carried as described in the Methods (Figure 1). The application of HP significantly increases the density of cells (Figure 2) after 1 week in OM compared to the static condition $\left(\mathrm{p}=10^{-5}\right)$, but the increase was not significant after 2 weeks $(p=0.07)$. Conversely, in $\mathrm{CM}$ a non-significant increase in the density of cells was observed with applied HP after 1 week $(p=0.21)$ and 2 weeks $(p=0.92)$. Importantly, we also observed a significant increase when incubated in OM compared to CM after 1 week of HP stimulation $(p=0.02)$. After 2 weeks of HP stimulation, samples cultured in OM exhibited a similar density to samples cultured in $\mathrm{CM}(\mathrm{p}=0.23)$. The results indicate that cell density increases more rapidly in the first week of stimulation in OM compared to CM media but that by two weeks the cell densities become equal. No significant difference was observed in the static cases after 1 or 2 weeks ( $p=0.99$ in both cases).

\section{Alkaline phosphatase activity assay}

The stimulation with cyclic HP significantly increased the ALP activity (Figure 3 ) in scaffolds incubated in OM after 1 and 2 weeks compared to static condition ( $p=4 \times 10^{-8}$ in both cases). A similar effect was observed in CM after 1 and 2 weeks ( $p=0.03$ and $p=5 \times 10^{-8}$ respectively). However, the incubation in OM significantly increased ALP activity when HP is applied compared to incubation in $\mathrm{CM}$, after 1 week $\left(\mathrm{p}<10^{-8}\right)$ but was not significantly different after 2 weeks $(p=0.99)$. Consistent with the cell density data the HP-driven increases in ALP activity are only observed during the first week and equalize by the second week of culture. In the absence of HP, the choice of incubation media did not significantly change the ALP activity after 1 or 2 weeks ( $p=0.25$ and $p=0.08$ respectively).

\section{Alizarin red S staining and mineral deposit quantification}

The application of cyclic HP significantly increased mineral deposition (Figure 4) for samples incubated in OM compared to static condition after 1 week and 2 weeks $\left(p=2 \times 10^{-7}\right.$ and $p=2 \times 10^{-8}$ respectively. Similarly in samples cultured in CM, cyclic HP significantly increased mineral deposition after 1 week and 2 weeks $\left(p=1 \times 10^{-6}\right.$ and $p=2 \times 10^{-8}$ respectively). Moreover, the incubation in OM significantly increased mineral deposition when HP is applied compared to incubation in CM, after 1 week $\left(p=2 \times 10^{-4}\right)$, but was not significant after 2 weeks $(p=0.99)$. These results are again consistent with the findings from assays of cell density and ALP activity. Under static conditions mineralization still occurred in $\mathrm{OM}$ as expected and was significantly increased compared to CM after 1 week $\left(p=10^{-3}\right)$, but was not significant after 2 weeks $(p=0.75)$. 


\section{Discussion}

Cells utilize a variety of mechanisms to sense and respond to a variety of mechanical stimuli (Vining and Mooney, 2017). Mechanical stimuli are known to affect cell differentiation, tissue regeneration, cytokines and protein expression and proliferation (Martino et al., 2018; Vining and Mooney, 2017). Thus, adequate representation of physical environment is critical in implant design (Campana et al., 2014; Gao et al., 2019; Nukavarapu et al., 2015; Parikh, 2002; Wang and Yeung, 2017). Bones are subjected to constant mechanical stresses and adapt through remodeling process (Bonewald and Johnson, 2008). In vivo, HP stimulates bone cells and impacts cell differentiation, maker expression and mineralisation (Henstock et al., 2013; Huang and Ogawa, 2012; Reinwald and El Haj, 2018). Plant-derived cellulose scaffolds are an emerging biomaterial in BTE (Lee et al., 2019), and therefore it is of interest to understand their performance under the mechanical conditions that are found in vivo (Gao et al., 2019; Nukavarapu et al., 2015). To our knowledge, cyclic HP effect of pre-osteoblasts seeded on plant-derived scaffolds have not yet been studied. Cellulose biomaterials derived from plant tissues have shown promising results in vitro and in vivo for targeted tissue engineering (Hickey et al., 2018; Modulevsky et al., 2016, 2014) and have been used to host osteoblastic differentiation (Lee et al., 2019). In addition, apple-derived cellulose scaffolds exhibit similar morphological characteristics to trabecular bone and were previously used for BTE applications (Lee et al., 2019).

In this study, we replicated the mechanical stimuli present during human locomotion by measuring the impact of differentiation markers in cellulose scaffolds seeded with pre-osteoblast cells. External pressure was applied on the scaffolds in similar magnitude of the lacunacanaliculi network with a frequency mimicking human locomotion (Henstock et al., 2013; Zhang et al., 1998). After proliferation, our scaffolds were either cultured in standard culture media, or in osteogenic-inducing differentiation media, with or without high pressure stimulation. Other groups utilizing similar cell lines (Gardinier et al., 2014, 2009), bone marrow skeletal stem cells (Reinwald and El Haj, 2018; Stavenschi et al., 2018; Zhao et al., 2015) or ex-vivo chick femur (Henstock et al., 2013) have also studied the effects if cyclic HP on either 2D surfaces, biomaterial meshes or ex vivo bones. In general, our results show a sensitivity ot HP during the early differentiation of the cells during the first week of stimulation. This sensitivity largely subsides during the second week of culture.

Cell counting revealed that the application of HP enhances MC3T3-E1 proliferation when cultured in OM or CM. Consistent with our work, are the results from previous studies in which metabolic activity has also been shown to be upregulated by mechanical stimulation in comparison to non-stimulated samples (Reinwald and El Haj, 2018). Moreover, it was shown that the application of HP accelerates cell proliferation through upregulated cell cycle initiation (Zhao et al., 2015). Similarly, reports have shown that physical stimulation of MC3T3-E1 cells induced expression of paracrine factors that leads enhancement of cell proliferation (Stavenschi et al., 2018). Importantly, when cultured in OM, cell density increases more rapidly with HP compared to CM during the first week of culture, but the cell densities equalize by the end of the second week. This data is consistent with previous reports of a time-dependent increase in cell number when cultured in OM (Quarles et al., 1992). Similarly, no significant difference between incubation of MC3T3-E1 cells in similar OM after 2 weeks (Hong et al., 2010). Our findings 
corroborate these studies and further suggest that the application of HP influences the replication rate at early stages of stimulation for samples cultured in OM.

Finally, ALP is an important enzyme expressed in the early stages of osteoblastic differentiation (Golub and Boesze-Battaglia, 2007). Our results indicate that the application of cyclic HP significantly increases ALP activity, compared to the static case. These findings are consistent with other studies on more conventional scaffolds (Reinwald and EI Haj, 2018). For example, a significant increase in ALP activity was also reported after the incubation of scaffolds in osteogenic-inducing differentiation media, similarly to reports on 2D culture systems (Hong et al., 2010; Quarles et al., 1992). The application of HP significantly increased the mineral content in the scaffolds after 1 week and 2 weeks of stimulation, in both types of incubation media. Other groups have shown that a cyclic $300 \mathrm{kPa}$ pressure at $2 \mathrm{~Hz}$ frequency on human BMSCs promoted significant mineral deposition (Stavenschi et al., 2018). The increase in mineral deposition also noted in ex vivo bone samples, with similar HP force application (Henstock et al., 2013). Furthermore, the incubation in OM increased the mineral content in the scaffolds, which is consistent with other studies (Hong et al., 2010; Quarles et al., 1992). Along with ALP expression, mineral content expression further confirms the ongoing differentiation of MC3T3-E1 onto osteoblast, either by applied HP, chemically (induction in OM) or a combination of both.

\section{Conclusion}

Plant-derived scaffolds have demonstrated their efficiency for tissue engineering applications (Fontana et al., 2017; Gershlak et al., 2017; Hickey et al., 2018; Lee et al., 2019; Modulevsky et al., 2016, 2014). The novelty, widespread availability, ease of use makes an interesting alternative to autografts, xenografts and synthetics implants. However, it has remined poorly understood how mechanosensitive pathways in bone precursor cells are impacted by being cultured in plant-derived cellulosic biomaterials. Previous studies have not included these phenomena. In this study, mechanical stimulation of cell-seeded scaffolds and the subsequent cellular response allowed us to understand the implants mechanosensitive behaviors in these novel scaffolds. As bone tissues are highly dynamic environments, relevant forces should be considered in the implant design. Importantly, this work provides the early evidence that boneprecursor cells appear to possess intact mechanosensing and mechanotransduction pathways when cultured on plant-derived scaffolds in a mechanically active environment. The results obtained here are consistent with data on traditional scaffold choices. The results reveal that application of cyclic HP, in combination with OM, leads to an increase in the number of cells, ALP activity and mineralization over time. These results combined with past in vitro and in vivo studies using apple-derived scaffold biomaterials demonstrate their potential in BTE applications.

\section{Acknowledgements}

This work was supported by a Discovery Grant from the Natural Sciences and Engineering Research Council of Canada (NSERC) and a grant from the Li Ka Shing Foundation. 


\section{References}

Andrzejowski, P., Giannoudis, P. V., 2019. The "diamond concept" for long bone non-union management. J. Orthop. Traumatol. https://doi.org/10.1186/s10195-019-0528-0

Balasundari, R., Bishi, D.K., Mathapati, S., Naser, S.B., Cherian, K.M., Guhathakurta, S., 2012. Nanocoated botanical scaffold in salvage for human tissue regeneration. J. Biomater. Tissue Eng. 2, 330-335. https://doi.org/10.1166/jbt.2012.1058

Bonewald, L.F., Johnson, M.L., 2008. Osteocytes, mechanosensing and Wnt signaling. Bone. https://doi.org/10.1016/j.bone.2007.12.224

Brunelli, M., Perrault, C., Lacroix, D., 2019. A Review of Bioreactors and Mechanical Stimuli. Springer, Singapore, pp. 1-22. https://doi.org/10.1007/978-981-10-8075-3_1

Campana, V., Milano, G., Pagano, E., Barba, M., Cicione, C., Salonna, G., Lattanzi, W., Logroscino, G., 2014. Bone substitutes in orthopaedic surgery: from basic science to clinical practice. J. Mater. Sci. Mater. Med. 25, 2445-2461. https://doi.org/10.1007/s10856014-5240-2

Di Martino, A., Sittinger, M., Risbud, M. V., 2005. Chitosan: A versatile biopolymer for orthopaedic tissue-engineering. Biomaterials. https://doi.org/10.1016/j.biomaterials.2005.03.016

Fernández, R.F., Bucchi, C., Navarro, P., Beltrán, V., Borie, E., 2015. Bone grafts utilized in dentistry: an analysis of patients' preferences. BMC Med. Ethics 16, 71. https://doi.org/10.1186/s12910-015-0044-6

Fontana, G., Gershlak, J., Adamski, M., Lee, J.-S., Matsumoto, S., Le, H.D., Binder, B., Wirth, J., Gaudette, G., Murphy, W.L., 2017. Biofunctionalized Plants as Diverse Biomaterials for Human Cell Culture. Adv. Healthc. Mater. 6, 1601225. https://doi.org/10.1002/adhm.201601225

Gao, X., Fraulob, M., Haïat, G., 2019. Biomechanical behaviours of the bone-implant interface: A review. J. R. Soc. Interface 16, 20190259. https://doi.org/10.1098/rsif.2019.0259

Gardinier, J.D., Gangadharan, V., Wang, L., Duncan, R.L., 2014. Hydraulic pressure during fluid flow regulates purinergic signaling and cytoskeleton organization of osteoblasts. Cell. Mol. Bioeng. 7, 266-277. https://doi.org/10.1007/s12195-014-0329-8

Gardinier, J.D., Majumdar, S., Duncan, R.L., Wang, L., 2009. Cyclic hydraulic pressure and fluid flow differentially modulate cytoskeleton re-organization in MC3T3 osteoblasts. Cell. Mol. Bioeng. 2, 133-143. https://doi.org/10.1007/s12195-008-0038-2

Gershlak, J.R., Hernandez, S., Fontana, G., Perreault, L.R., Hansen, K.J., Larson, S.A., Binder, B.Y.K., Dolivo, D.M., Yang, T., Dominko, T., Rolle, M.W., Weathers, P.J., Medina-Bolivar, F., Cramer, C.L., Murphy, W.L., Gaudette, G.R., 2017. Crossing kingdoms: Using decellularized plants as perfusable tissue engineering scaffolds. Biomaterials 125, 13-22. https://doi.org/10.1016/J.BIOMATERIALS.2017.02.011

Golub, E.E., Boesze-Battaglia, K., 2007. The role of alkaline phosphatase in mineralization. Curr. Opin. Orthop. 18, 444-448. https://doi.org/10.1097/BCO.0b013e3282630851

Gregory, C.A., Gunn, W.G., Peister, A., Prockop, D.J., 2004. An Alizarin red-based assay of mineralization by adherent cells in culture: Comparison with cetylpyridinium chloride 
extraction. Anal. Biochem. 329, 77-84. https://doi.org/10.1016/j.ab.2004.02.002

Henstock, J.R., Rotherham, M., Rose, J.B., El Haj, A.J., 2013. Cyclic hydrostatic pressure stimulates enhanced bone development in the foetal chick femur in vitro. Bone 53, 468477. https://doi.org/10.1016/j.bone.2013.01.010

Hickey, R.J., Modulevsky, D.J., Cuerrier, C.M., Pelling, A.E., 2018. Customizing the Shape and Microenvironment Biochemistry of Biocompatible Macroscopic Plant-Derived Cellulose Scaffolds. ACS Biomater. Sci. Eng. 4, 3726-3736. https://doi.org/10.1021/acsbiomaterials.8b00178

Hickey, R.J., Pelling, A.E., 2019. Cellulose biomaterials for tissue engineering. Front. Bioeng. Biotechnol. https://doi.org/10.3389/fbioe.2019.00045

Hong, D., Chen, H.X., Yu, H.Q., Liang, Y., Wang, C., Lian, Q.Q., Deng, H.T., Ge, R.S., 2010. Morphological and proteomic analysis of early stage of osteoblast differentiation in osteoblastic progenitor cells. Exp. Cell Res. 316, 2291-2300. https://doi.org/10.1016/j.yexcr.2010.05.011

Huang, C., Ogawa, R., 2012. Effect of Hydrostatic Pressure on Bone Regeneration Using Human Mesenchymal Stem Cells. Tissue Eng. Part A 18, 2106-2113. https://doi.org/10.1089/ten.tea.2012.0064

Lee, J., Jung, H., Park, N., Park, S.H., Ju, J.H., 2019. Induced Osteogenesis in Plants Decellularized Scaffolds. Sci. Rep. 9, 1-10. https://doi.org/10.1038/s41598-019-56651-0

Lee, J.C., Volpicelli, E.J., 2017. Bioinspired Collagen Scaffolds in Cranial Bone Regeneration: From Bedside to Bench. Adv. Healthc. Mater. https://doi.org/10.1002/adhm.201700232

Liu, C., Zhao, Y., Cheung, W.Y., Gandhi, R., Wang, L., You, L., 2010. Effects of cyclic hydraulic pressure on osteocytes. Bone 46, 1449-1456. https://doi.org/10.1016/j.bone.2010.02.006

Martino, F., Perestrelo, A.R., Vinarský, V., Pagliari, S., Forte, G., 2018. Cellular mechanotransduction: From tension to function. Front. Physiol. https://doi.org/10.3389/fphys.2018.00824

Modulevsky, D.J., Cuerrier, C.M., Pelling, A.E., 2016. Biocompatibility of Subcutaneously Implanted Plant-Derived Cellulose Biomaterials. PLoS One 11, e0157894. https://doi.org/10.1371/journal.pone.0157894

Modulevsky, D.J., Lefebvre, C., Haase, K., Al-Rekabi, Z., Pelling, A.E., 2014. Apple derived cellulose scaffolds for 3D mammalian cell culture. PLoS One 9, e97835. https://doi.org/10.1371/journal.pone.0097835

Morrell, K.C., Hodge, W.A., Krebs, D.E., Mann, R.W., 2005. Corroboration of in vivo cartilage pressures with implications for synovial joint tribology and osteoarthritis causation. Proc. Natl. Acad. Sci. U. S. A. 102, 14819-14824. https://doi.org/10.1073/pnas.0507117102

Nukavarapu, S.P., Freeman, J.W., Laurencin, C.T., 2015. Regenerative Engineering of Musculoskeletal Tissues and Interfaces, Regenerative Engineering of Musculoskeletal Tissues and Interfaces. Elsevier Inc. https://doi.org/10.1016/C2014-0-02826-2

Parikh, S.N., 2002. Bone graft substitutes: past, present, future. J. Postgrad. Med. 48, 142-148.

Pörtner, R., Nagel-Heyer, S., Goepfert, C., Adamietz, P., Meenen, N.M., 2005. Bioreactor design for tissue engineering. J. Biosci. Bioeng. https://doi.org/10.1263/jbb.100.235 
Quarles, L.D., Yohay, D.A., Lever, L.W., Caton, R., Wenstrup, R.J., 1992. Distinct proliferative and differentiated stages of murine MC3T3-E1 cells in culture: An in vitro model of osteoblast development. J. Bone Miner. Res. 7, 683-692. https://doi.org/10.1002/jbmr.5650070613

Reinwald, Y., El Haj, A.J., 2018. Hydrostatic pressure in combination with topographical cues affects the fate of bone marrow-derived human mesenchymal stem cells for bone tissue regeneration. J. Biomed. Mater. Res. - Part A 106, 629-640. https://doi.org/10.1002/jbm.a.36267

Reinwald, Y., Leonard, K.H.L., Henstock, J.R., Whiteley, J.P., Osborne, J.M., Waters, S.L., Levesque, P., El Haj, A.J., 2015. Evaluation of the growth environment of a hydrostatic force bioreactor for preconditioning of tissue-engineered constructs. Tissue Eng. - Part C Methods 21, 1-14. https://doi.org/10.1089/ten.tec.2013.0476

Sakkas, A., Wilde, F., Heufelder, M., Winter, K., Schramm, A., 2017. Autogenous bone grafts in oral implantology-is it still a "gold standard"? A consecutive review of 279 patients with 456 clinical procedures. Int. J. Implant Dent. 3. https://doi.org/10.1186/s40729-017-0084-4

Schindelin, J., Arganda-Carreras, I., Frise, E., Kaynig, V., Longair, M., Pietzsch, T., Preibisch, S., Rueden, C., Saalfeld, S., Schmid, B., Tinevez, J.-Y., White, D.J., Hartenstein, V., Eliceiri, K., Tomancak, P., Cardona, A., 2012. Fiji: an open-source platform for biologicalimage analysis. Nat. Methods 9, 676-682. https://doi.org/10.1038/nmeth.2019

Stavenschi, E., Corrigan, M.A., Johnson, G.P., Riffault, M., Hoey, D.A., 2018. Physiological cyclic hydrostatic pressure induces osteogenic lineage commitment of human bone marrow stem cells: A systematic study. Stem Cell Res. Ther. 9. https://doi.org/10.1186/s13287018-1025-8

Torgbo, S., Sukyai, P., 2018. Bacterial cellulose-based scaffold materials for bone tissue engineering. Appl. Mater. Today 11, 34-49. https://doi.org/10.1016/J.APMT.2018.01.004

Vining, K.H., Mooney, D.J., 2017. Mechanical forces direct stem cell behaviour in development and regeneration. Nat. Rev. Mol. Cell Biol. https://doi.org/10.1038/nrm.2017.108

Wang, W., Yeung, K.W.K., 2017. Bone grafts and biomaterials substitutes for bone defect repair: A review. Bioact. Mater. https://doi.org/10.1016/j.bioactmat.2017.05.007

Wei, S., Ma, J.X., Xu, L., Gu, X.S., Ma, X.L., 2020. Biodegradable materials for bone defect repair. Mil. Med. Res. https://doi.org/10.1186/s40779-020-00280-6

Zhang, D., Weinbaum, S., Cowin, S.C., 1998. Estimates of the peak pressures in bone pore water. J. Biomech. Eng. 120, 697-703. https://doi.org/10.1115/1.2834881

Zhao, Y.H., Lv, X., Liu, Y.L., Zhao, Y., Li, Q., Chen, Y.J., Zhang, M., 2015. Hydrostatic pressure promotes the proliferation and osteogenic/chondrogenic differentiation of mesenchymal stem cells: The roles of RhoA and Rac1. Stem Cell Res. 14, 283-296. https://doi.org/10.1016/j.scr.2015.02.006 


\section{FIGURE LEGENDS}

Figure 1: (A) Cyclic hydrostatic pressure device schematics. Hydrostatic pressure was applied by modulating the pressure in the gas phase above the culture wells in a custom-build pressure chamber. Air from incubator atmosphere was compressed using a compressor and injected in the pressure chamber using solenoid valves. (B) Experimental conditions. After 1 week of proliferation, cyclic hydrostatic pressure stimulation was applied during 1 hour per day, for up to 2 weeks at a frequency $1 \mathrm{~Hz}$, oscillating between 0 and $280 \mathrm{kPa}$ with respect to ambient pressure. The samples were removed from the pressure chamber after each cycle and kept at ambient pressure between the stimulation phases.

Figure 2: (A) Representative confocal laser scanning microscope image showing seeded cells scaffolds (scale bar $=100 \mu \mathrm{m}-$ applies to all). The scaffolds were stained for cellulose (red) and for cell nuclei (blue). (B) Cellular density after 1 week or 2 weeks of stimulation. Statistical significance ( ${ }^{*}$ indicates $p<0.05$ ) was determined using a one-way ANOVA and Tukey post-hoc tests. Data are presented as means \pm S.E.M. of three replicate samples per condition, with three areas per sample.

Figure 3: Alkaline phosphatase (ALP) activity after 1 week or 2 weeks of stimulation. Statistical significance ( ${ }^{*}$ indicates $p<0.05$ ) was determined using a one-way ANOVA and Tukey post-hoc tests. Data are presented as means \pm S.E.M. of three replicate samples per condition.

Figure 4: Mineral deposit quantification with Alizarin Red S (ARS) staining after 1 week or 2 weeks of stimulation. Statistical significance $\left({ }^{*}\right.$ indicates $\left.p<0.05\right)$ was determined using a oneway ANOVA and Tukey post-hoc tests. Data are presented as means \pm S.E.M. of three replicate samples per condition. 


\section{Figure 1}

A

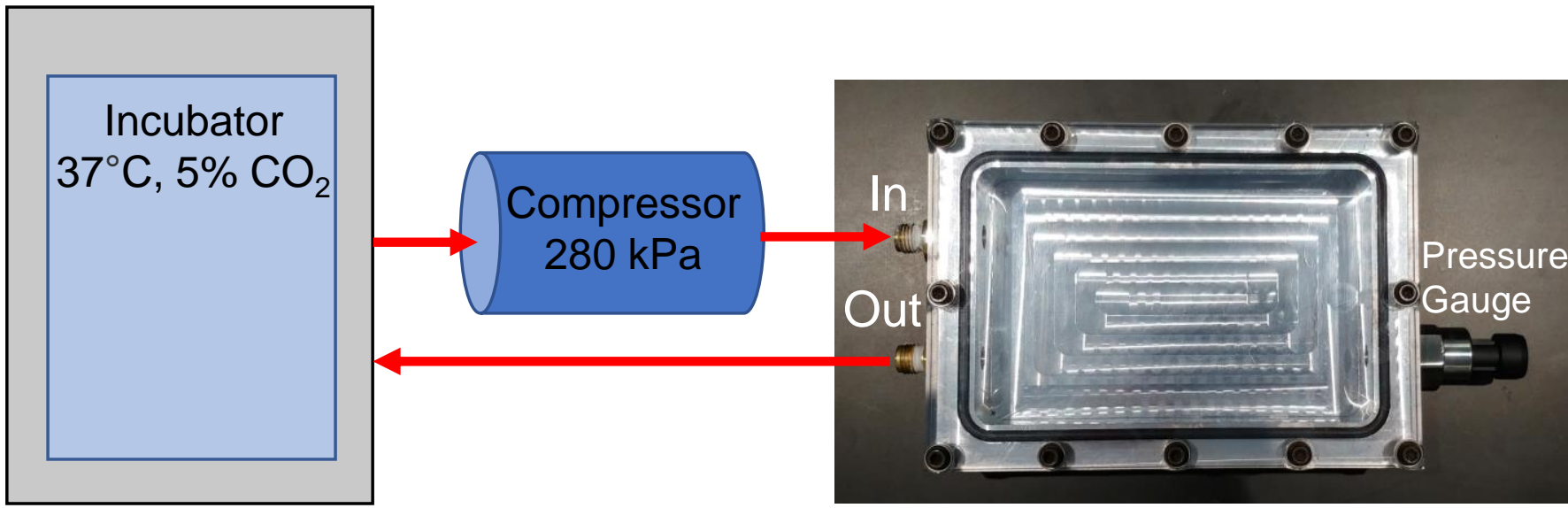

B

Proliferation

Stimulation

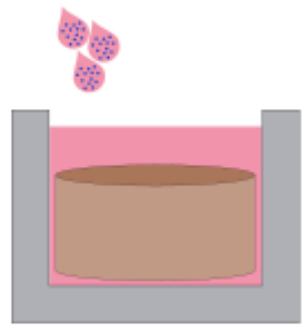

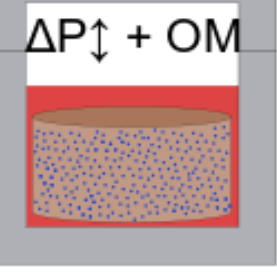

$\mathrm{OM}$

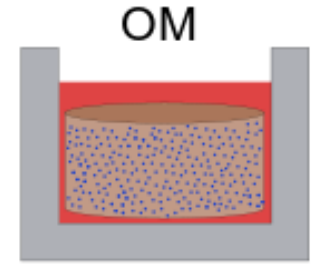

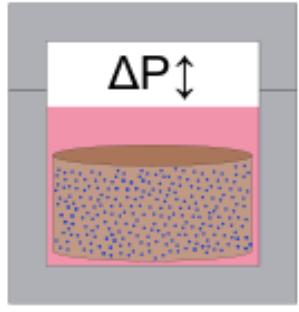

Control

$=$ Culture media

$=$ Osteogenic media

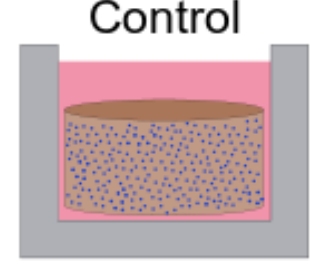




\section{Figure 2}

OM-HP

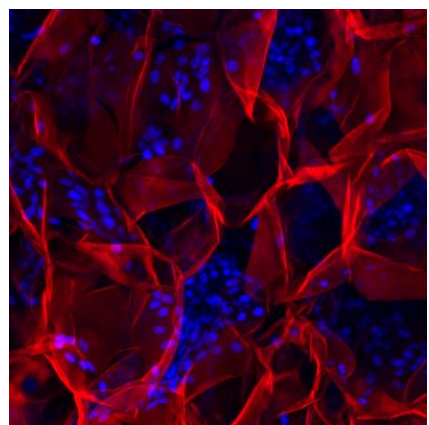

CM-HP

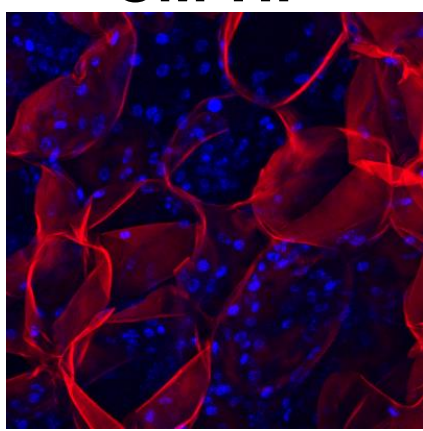

OM-CTRL

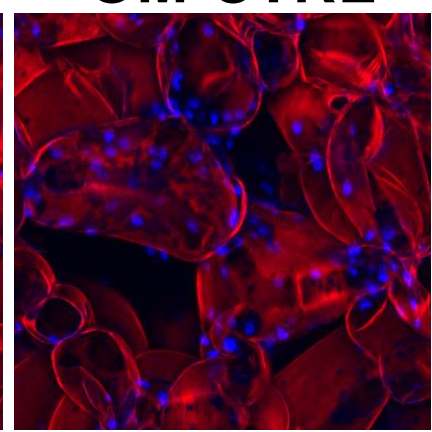

CM-CTRL

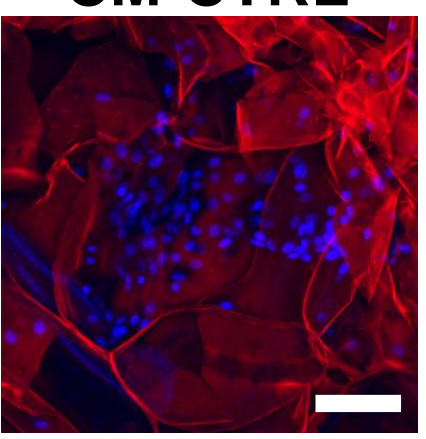

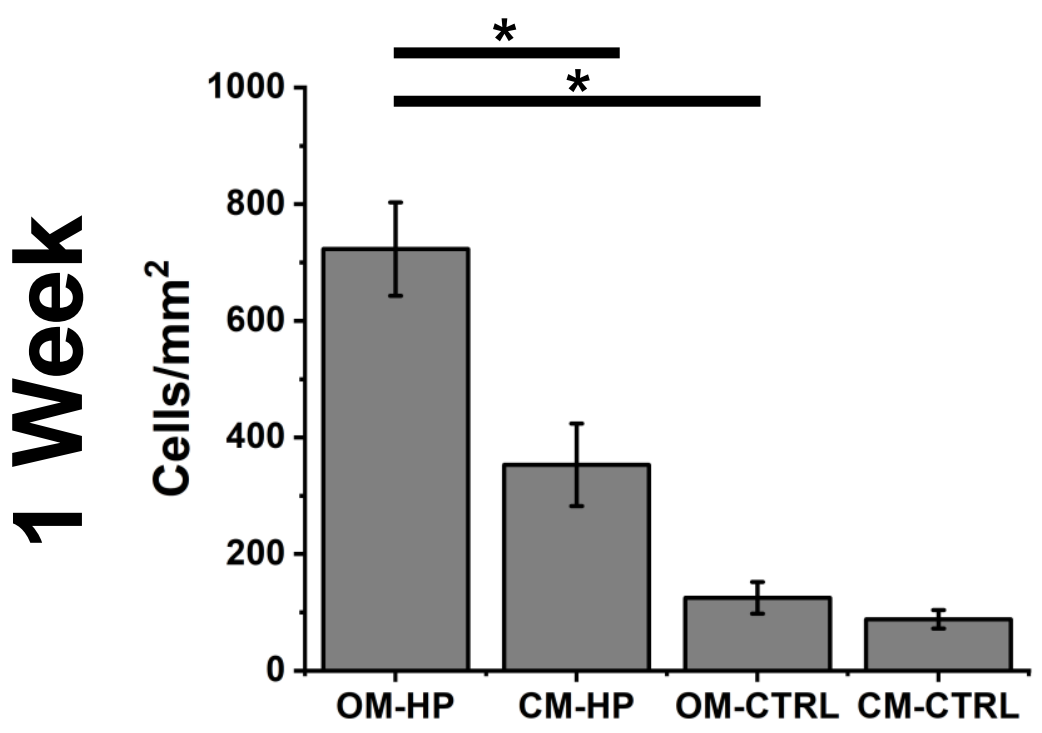
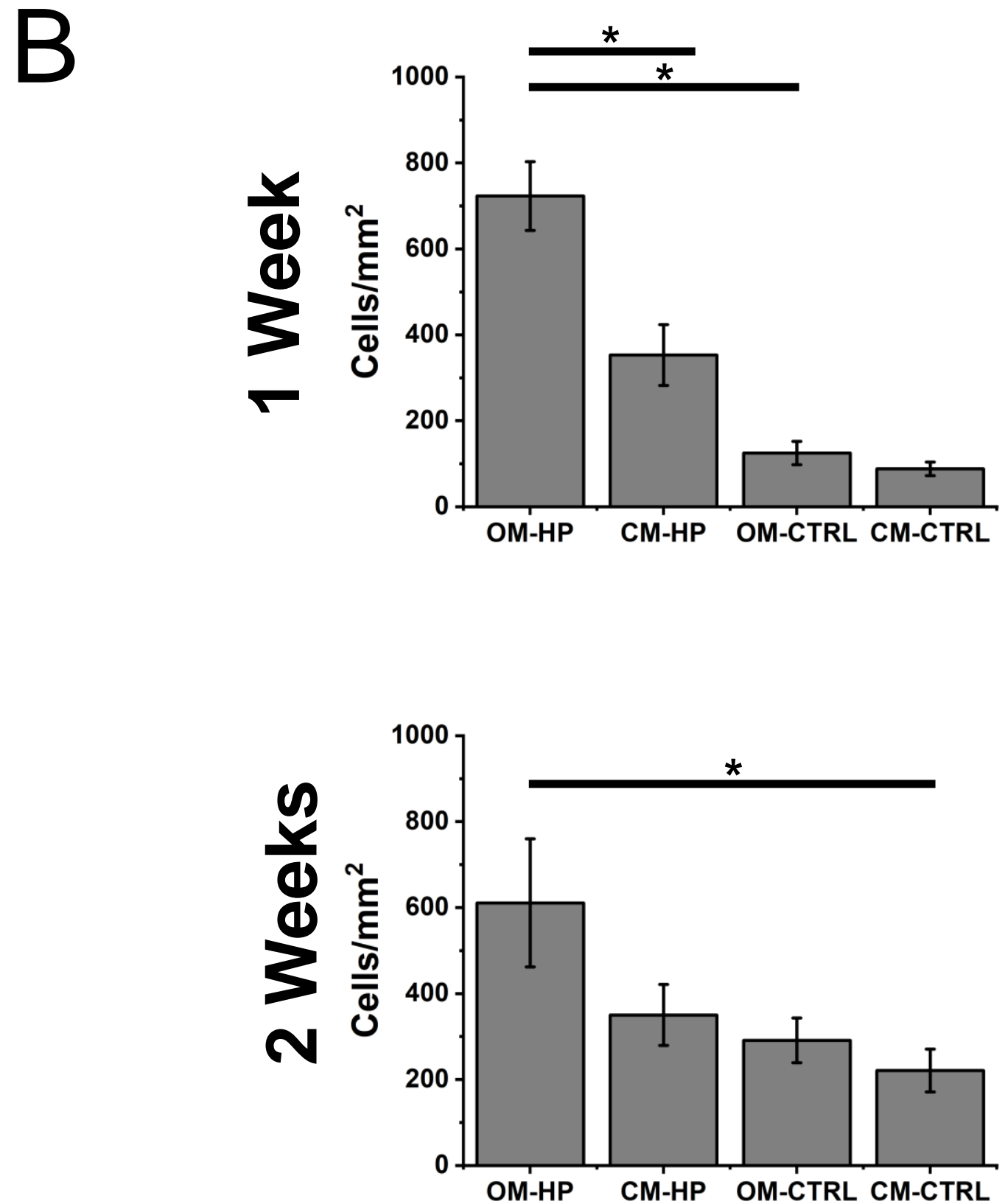


\section{Figure 3}
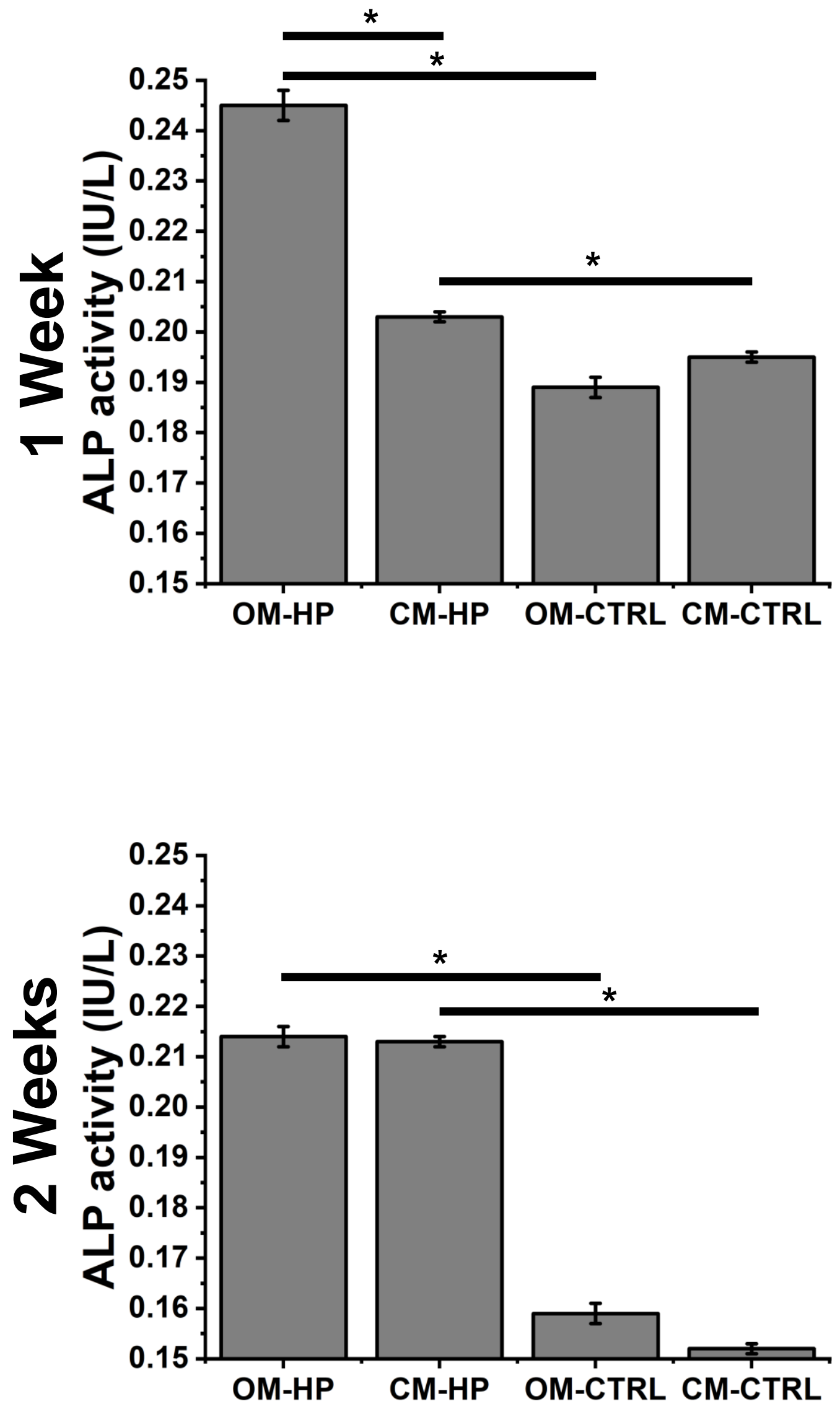


\section{Figure 4}
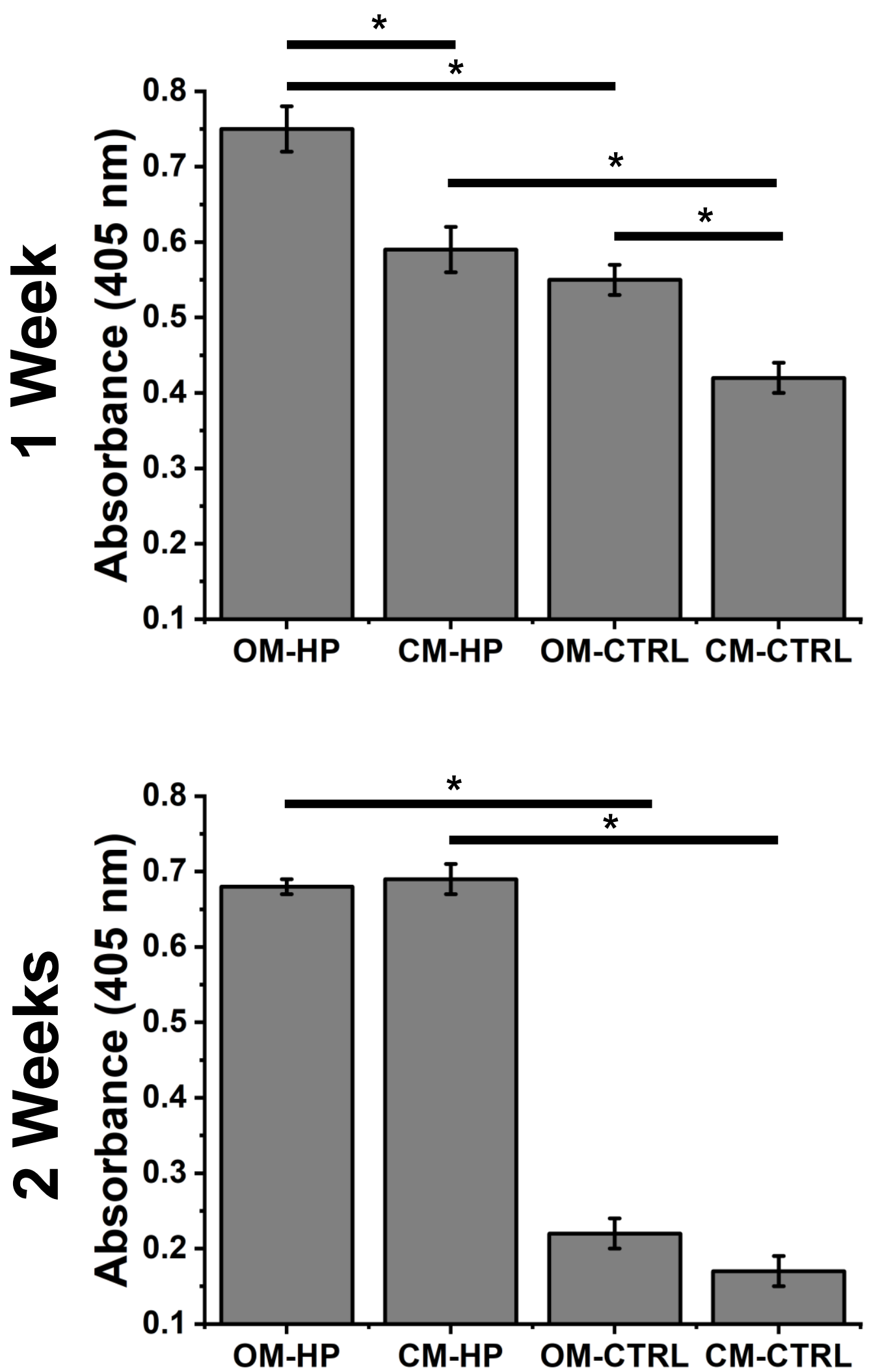